\title{
La historia, las fuentes orales y la enseñanza: teoría y práctica
}

\author{
María Gloria Nuñez Pérez
}

Este texto trata de mostrar algunos de los principios que señalan la conveniencia de la introducción de las fuentes orales en la enseñanza de la historia y apunta algunas posibilidades prácticas en orden a su inserción.

\section{PLANTEAMIENTO TEÓRICO}

Si bien todo lo referido al pasado del hombre cae dentro del término historia, también el mismo concepto define el conjunto de hechos que un investigador recoge para hacer "su" historia ${ }^{1}$, aunque, en el mundo académico nacional e internacional, la palabra historiografía sirve asimismo para nombrar el conjunto de obras realizadas con la finalidad de historiar $^{2}$. El hombre ha ido elaborando diferentes modalidades de historia en la medida que elegía unos determinados acontecimientos materiales o espirituales, unos métodos y unas finalidades, lo cual se relaciona, como lo muestra la historia de la historiografía, con factores surgidos de la propia personalidad del historiador y de su peculiar entorno cultural,

\footnotetext{
' Sobre la ambigüedad del término que, en la mayoría de las lenguas cultas, sirve para designar ambos campos: MaRRou, Henri-Irénée, El conocimiento histórico. Barcelona, Labor, 1968, págs. 31-34.

${ }^{2}$ Una explicitación del término historiografia e historia de la historiografia en SANCHEZ ALonso, Benito, “El concepto de historiografía española", Hispania, 11. Madrid, 1943, págs. 179-194; CaRBonelL, Charles-Oliver. "Pour une histoire de I'historiographie", Storia della storiografia, 1. Milán, 1982, pág. 7-25.
} 
social y económico. Como todos sabemos cada época reescribe la historia, lo cual no impide la existencia de obras que atraviesan los siglos en función de su aportación significativa e imperecedera al conocimiento del ayer.

Las variadas formas de edificar el discurso histórico tiene cierta similitud con las que adoptan otros tipos de saberes humanos, tales como, por ejemplo, la ciencias experimentales. Aludiré seguidamente a ello porque podría parangonarse con la inclusión de las fuentes orales en la historiografía reciente, motivo que, entre otros, posibilita su utilización en la didáctica de la historia.

En el siglo $x \mid x$, el historiador alemán Leopold Von Ranke colocó la historia en el ámbito académico y científico al levantarla a partir del conocimiento de hechos convenientemente probados en fuentes documentales suficientemente contrastadas y criticadas ${ }^{3}$. Pero la acumulación de acontecimientos, sin la formulación de teorías o leyes generales, llevaron al desprestigio del llamado positivismo historicista. Se apuntó que el conocimiento histórico no podía ser llamado científico porque no era capaz de predecir, es decir, validarse o falsarse mediante la experimentación ${ }^{4}$. No obstante tal planteamiento fue puntualizado tras mostrar que los experimentos cruciales no son los que refutan las teorías científicas. Más bien brota el rechazo cuando la comunidad de expertos, la sociedad, adoptan nuevos paradigmas o modelos de problemas y soluciones que no pueden ser resueltos con las viejas concepciones, y que surgen en conexión con movimientos sociales y culturales de la época ${ }^{5}$. La comprobación de la sucesión temporal de paradigmas científicos, los cuales explican únicamente parte de la realidad, han acercado las ciencias llamadas naturales a las sociales. Por ejemplo, la física ha ido evolucionando, construyéndose con los modelos geométrico, mecánico, relativo/ cuántico, y en los últimos años ha surgido el paradigma llamado física de la complejidad. Dentro de éste, resulta imposible la predicción. $Y$ esto es asi tanto para el movimiento de los astros como para las reacciones químicas o las dinámicas económicas y sociales, pues en las condiciones

${ }^{3}$ Una defensa sinóptica del dicurso del historiador decimonónico en Fusı AlzPURUA, Juan Pablo, "Por una nueva historia: volver a Ranke", Perspectiva contemporánea. España siglo $x x, 1$. Madrid, octubre de 1988, págs. 153-154.

4 Sobre la cuestión: POPPER, Karl. La miseria del historicismo. Madrid, Taurus, 1961 (Londres, 1957).

5 Véase KHun, Thomas S., La esctructura de la revoluciones cientificas. México, FCE, 1982 (Chicago, 1962); LAKATOS, Imre. La metodología de los programas de investigación cientifica, Madrid, 1983 (Londres, 1978); FEYERABEnd, Paul K., Contra el método. Barcelona, Ariel, 1974 (Minneapolis, 1970). 
de partida siempre hay una incertidumbre generada por los propios sistemas de medición, los cuales producen en el tiempo una desviación de las trayectorias calculadas. Ha surgido la complejidad dinámica del caos determinista. Aparece en los casos de dinámica no lineal, es decir, en el comportamiento más común en la naturaleza. A la vez se ha visto la complejidad estructural que precipita a tierra el construccionismo 0 , 10 que es lo mismo, la elaboración conceptual del comportamiento de un objeto complicado a partir del conocimiento de sus componentes. Cada nivel de complejidad contiene nuevas propiedades ${ }^{6}$.

Ahora la historia también está dentro de la corriente de complejidad, de la enorme variabilidad de contextos y circunstancias, de la imposibilidad de definir con rigurosidad extrema un conjunto de supuestos. Pero a la vez la historia se ha hecho más múltiple que nunca, lanzándose a entender nuevos mecanismos, dentro de la totalidad inabarcable. Adopta numerosos puntos de vista para interrogar al pasado. En este sentido, pone los ojos en diferentes espacios: la nación, la región, la localidad, la familia y llega hasta las historias de vida o las biografías. Escoge aspectos políticos, económicos, sociales, antropológicos o culturales que a su vez adquieren multitud de opciones de investigación. Por ejemplo: determinadas élites políticas, una categoria social (las mujeres), la vida privada, un tipo de mentalidad, algún movimiento popular. Decide en qué tiempo va a investigar: en el corto del suceso, en el de la coyuntura, en el más largo de la estructura y ello, lo mismo dentro de un remoto pasado que dentro del tiempo reciente. Selecciona una metodología y unos modelos a aplicar (cuantitativos, estructurales, funcionales, marxistas...) Determina una forma para su discurso, bien sea narrativo lineal o multilineal. Luego opta por indagar entre los innumerables tipos de fuentes que recogen las palabras, los sonidos, las imágenes o cualquier resto material o espiritual del pasado ?.

¿Cuál de estos puntos de vista es más válido o, mejor, el que merecería la pena trasmitir en una didáctica de la historia? Primero, evidentemente, habría que tener en cuenta los niveles educativos, la edad o el

\footnotetext{
6 Una sintesis del tema en ARECH, Tito. "Caos y complejidad", El Pais Liber 1. Madrid, 11 de octubre de 1989, págs. 14-15.

7 Sobre la formas de hacer la historia: LE GOFF, Jaques (dir.) Diccionario del saber moderno: la nueva historia. Bilbao, Mensajero, 1988 (París, 1988); CARBonelL, CharlesOliver, La historiografia. México, FCE, 1986 (París, 1981); Stone, Lewis, El pasado y el presente. México, FCE, 1986; HimmelfarB, Gertrude, The New history and the old Critical essays and reappraisals. Cambridge, Massachusetts, London Harvard University Press, 1987; Dosse, François, L'histoire en miettes. Des “Annales" à la nouvelle histoire. Paris, Edition La Découverte, 1987.
} 
grado de especialización de los alumnos en tanto que cada grupo asimilaría en diferentes grados la complejidad histórica. Pero, en general, creo en la conveniencia de mostrar a las nuevas generaciones los diferentes modos de hacer la historia, siempre que aparezcan fundamentados en fuentes varificables y explicitado de alguna manera comprensible qué objetivos persigue, qué métodos utiliza y cuales son los resultados obtenidos. Independientemente de considerar el marco cronológico y político como un primer referente a la hora de ensamblar diversos aspectos de lo histórico, interesaría revelar las formas de historiografía que han encontrado nuevas hipótesis metodologias, fuentes o han ordenado y secuenciado más claramente el pasado, de tal manera que éste se haya ensanchado significativamente.

Uno de los períodos de estudio incorporados últimamente a la historia corresponde al tiempo actual. La construcción de éste a partir de fuentes orales también constituye una innovación, como asimismo lo es la inserción de esas fuentes en calidad de recurso didáctico.

Resulta comunmente sabido que los historiadores manejaron tales testimonios desde la antigüedad, aunque la legitimidad otorgada al documento escrito en el siglo XIX, pues se constituía como la prueba de credibilidad del relato histórico, ocasionó el abandono de aquéllos. Pero a mediados de la presente centuria, la historiografía occidental comienza a emplearlos al formularse unas hipótesis y cuestiones que los documentos escritos no conseguian responder. El ejemplo de la utilización del material oral por parte de la sociología y antropología, junto con la posibilidad de grabar magnéticamente la verosimilitud del testimonio, influyeron en la adopción de esas fuentes por los investigadores. Allí buscaron las experiencias reales o imaginarias que del pasado tenian en la mente hombres y mujeres vivos. Se recurrió a ellas para indagar, por ejemplo, la historia de los grupos populares, marginados, clandestinos, minoritarios o étnicos; las élites; la vida cotidiana o privada; las mentalidades; los movimientos migratorios; los pueblos ágrafos. La aparición y crecimiento de las fuentes orales grabadas en cinta magnética ocasionó la apertura de los llamados archivos orales dedicados a custodiar y también promover la recogida de testimonios. Por otra parte, como cualquier innovación en todo saber, hubo reticencias hacia su legitimidad, problematizando, por ejemplo, la fiabilidad de la memoria, la subjetividad intensificada por el paso del tiempo, los mecanismos de autojustificación personal, la representatividad. Pero su validez va demostrándose paulatinamente, partiendo de la base de que todo documento humano, tanto escrito como hablado, adolece de esas insuficiencias sin que, por ello, los historiadores orales dejen de afirmar que el tratamiento y más aún la elaboración de 
la fuente, es decir, las entrevistas o los cuestionarios, requieren una metodología estricta y acotada respecto a su alcance y límites. También han señalado que, como cualquier otro documento, debía ser compulsado, constrastado y probado para que fuera fiable, además de completado con otro tipo de fuentes, para poder contrarrestar las respectivas subjetividades, alcanzar así cierta objetividad y lograr una construcción histórica capaz de saltar de lo individual a lo colectivo ${ }^{8}$.

En España, ya en los años setenta hubo alguna investigación realizada con fuentes orales, aunque es en la última década cuando ha crecido su número, orientándose hacia el estudio de la guerra civil, mundo obrero, represión franquista, guerrilleros y maquis, mujeres, vida cotidiana, etc. También se han celebrado congresos y jornadas monográficas alrededor de esas fuentes, y en simposios sobre épocas recientes ha habido comunicaciones realizadas principalmente a partir de ellas. Además se han constituido algunos centros para la recogida de material oral y hay en marcha proyectos de acopio de testimonios en torno a determinados centros de interés ${ }^{9}$.

${ }^{8}$ Las conocidas obras de Ph. Joutard, P. Thompson, J. Vansina, G. E. Evans, R. Fraser, etc. han puesto de manifiesto la validez de las fuentes orales para construir el pasado. Puede verse una síntesis sobre cuestiones de método en ALTED VIGIL. Alicia. "EI testimonio oral como fuente histórica (Aproximación metodológica)", en Perspectiva, contemporánea. España siglo xx. 1 Madrid, octubre de 1988, págs. 155-162. Sobre historiografía oral: HENIGE, David, Oral Historiography, London, Longman, 1982 y 1985 (2. ${ }^{a}$ ed.) Una muestra del recorrido apreciable de la utilización de las fuentes orales es la próxima celebración, entre el 29 de marzo y 1 de abril de 1990, de la "VII Conferencia Internacional de Historia Oral", en Essen (República Federal de Alemania). En 1980, el "XV Congreso Internacional de Ciencias Históricas" (Bucarest) las incorporó a sus sesiones.

${ }^{9}$ Sólo en calidad de muestra de investigaciones con diferente temática y utilizando fuentes orales: FrASER, Ronald. Recuérdalo tu y recuérdalo a los otros. Historia oral de la guerra civil española, 2 vol. Barcelona, Crítica, 1979; Monjo, Ana, y VEGA, Carme, Els traballadors i la guerra civil. Historia d'una industria catalana collectivitzada. Barcelona, Empuries, 1986; Sole I SABATE, Josep M., La repressió franquista a Catalunya, 1936-1953. Barcelona, Ediciones Sesenta y dos, 1985; Romeu, Fernanda. La agrupación guerrillera de Levante. Valencia, Institución Alfonso el Magnánimo, 1987; FolguerA, Pilar, Vida cotidiana en Madrid. Primer tercio del siglo xx a través de fuentes orales. Madrid, Comunidad de Madrid, 1987; VILANOVA. Mercedes, "El abstencionismo electoral y su relación con las fuerzas politicas en la provincia de Gerona durante la segunda república. Un ejemplo: La Escala", en Homenaje al profesor Reglá. Valencia, Universidad de Valencia, 1975; BoRDERIAS, Cristina. "Identidad femenina y cambio social en Barcelona entre 1920 y 1980", en VILA. NOVA, Mercedes (edits.). El poder en la sociedad. Historia y fuente oral. Barcelona, Antoni Bosh, 1986; Apalategul, J. M., Introducción a la historia oral a través de los "kontuzaha. rrak" (cuentos viejos) de la comunidad guipuzcoana de Ataum. Barcelona 1987.

También se han celebrado coloquios en España en torno a esas fuentes. Así, en Paima de Mallorca (abril de 1984). Al año siguiente la $\backsim V$ Conferencia Internacional de Historia Oral", tuvo su sede en Barcelona. Después el Seminario de Fuentes Orales (asociación cuyo objetivo consiste en promover la investigación histórica a partir de los testimonios 
Cómo las fuentes orales constituyen uno de los materiales para construir el conocimiento histórico, parece obvio su utilización en la didáctica y práctica de la investigación del pasado reciente. El segundo y tercer ciclo de la enseñanza superior constituyen los niveles propios donde abordar ese estudio ${ }^{10}$.

Pero aquí me referiré a su utilización en la didáctica de la historia, no de la investigación histórica, en el período actualmente correspondiente a los últimos cursos del bachillerato y primeros de enseñanza universi-

taria, cuando el conocimiento humano se presenta a los alumnos ya sistematizado y articulado en disciplinas. De ellas tienen que captar la base abstracta e iniciarse en la comprensión de los métodos de investigación.

Si hace unos años la didáctica estaba basada en la pedagogía por objetivos, la cual expresaba la pretensión educativa a conseguir en términos de conducta observable en los alumnos (teoría conductista de

hablados) organizó, junto con otras entidades culturales, varios simposios: el titulado «Diseño de Proyectos de Historia Oral», en la Facultad de Geografía-Historia de la Universidad Complutense de Madrid (29 a 30 de abril de 1988); el “Primer Encuentro de Fuentes Orales y Enseñanza de la Historia», reunido en el Centro Asociado de la Universidad Nacional de Educación a Distancia de Ávila (21 a 23 de abrl de 1989). Este año celebrará en el mismo lugar, en el mes de abril, las "Segundas Jornadas sobre Fuentes Orales e Investigación Histórica: Técnicas y Metodología".

Asimismo en congresos y jornadas sobre épocas recientes suelen presentarse comunicaciones basadas en este tipo de fuentes. Por ejemplo, citando alguno de los más próximos en el tiempo: "La oposición al régimen de Franco", celebrado en Madrid entre el 19 y 22 de octubre de 1988, organizado por el Departamento de Historia Contemporánea de la UNED; "Movimiento guerrillero de los años cuarenta", organizado por la Fundación de Investigaciones Marxistas y Ministerio de Cultura en Madrid, entre el 24 y 26 de noviembre de 1988, "Las mujeres en la guerra civil española", bajo la iniciativa del Archivo HistóricoSección Guerra Civil en Salamanca, durante los días 4 al 6 de octubre de 1989.

Existen, en nuestro pais, algunos centros que guardan documentación oral. Uno es el Archivo de Historia Oral del Institut Municipal d'Historia de Barcelona. Una información sobre sus fondos en Historia y fuente oral, 1. Barcelona, 1989, págs. 161-176. El Centro de Información y Documentación de Archivos (Madrid) lieva actualmente a cabo el proyecto de la Dirección de Archivos Estatales para recabar testimonios en torno al tema "El éxilio español en México". Aquéllos pasarán a formar parte de los fondos del Archivo Histórico Nacional-Sección Guerra Civil de Salamanca. Algunos departamentos de historia de las universidades españolas también efectúan cierto almacenaje de testimonios. Es el caso, por ejemplo, del Departamento de Historia Contemporánea de la UNED y del Laboratorio de Fuentes Orales de la Facultad de Geografía-Historia de Santiago de Compostela. También el citado Seminario de Fuentes Orales conserva testimonios grabados.

10 Ya se emplean en esos niveles. En el referido «Primer Encuentro de Fuentes Orales y Enseñanza de la Historia", hubo comunicaciones refiriendo algunas experiencias llevadas a cabo en la UNED, Universidad de Santiago de Compostela y Universidad de Salamanca. 
Skinner), actualmente es preceptivo considerar los descubrimientos de la psicologia cognitiva de la instrucción, también empírica, pero que además tiene en cuenta las teorias sobre el aprendizaje. Los modelos educativos aparecen fundamentados sobre todo en el análisis de contenidos en función de las capacidades de procesamiento cognitivo de los alumnos. Asimismo tratan de explicitar las estrategias de aprendizaje para lograr mejorar las destrezas académicas ". Los modelos señalan el gran número de variables que influyen en el aprendizaje. Entre las más útiles, por promover la enseñanza-aprendizaje, tenemos dentro del modelo cognoscitivo: el establecimiento de objetivos, el aprendizaje significativo, la organización del material a captar y asimilar mentalmente por medio de configuraciones globales, el conocimiento de los aprendizajes erróneos. Dentro del modelo neoconductista: la participación activa, el reforzamiento-premio como incremento en el proceso de aprender, la progresión de la dificultad, la generalización y discriminación para la adquisición de conceptos ${ }^{12}$.

En el diseño curricular de la asignatura de historia es donde corresponde integrar en principio las fuentes orales. El currículum partiría de considerar los principios epistemológicos y temáticos de la historia, junto con las características psicológicas, culturales y sociales del alumnado. Después establecería los objetivos generales, las estructuración y selección de los contenidos (hechos y conceptos, procedimientos y actitudes), la metodología didáctica y las formas de evaluación ${ }^{13}$.

"Algunas de las teorías son: la optimización del aprendizaje cuanto éste es significativo, es decir, la nueva información se relaciona con conocimientos ya acumulados en la memoria (Ausubel, Novak, Gowin); el aprendizaje generativo mediante el cual se aprende engarzando y uniendo lo nuevo con lo anteriormente acumulado en la mente (Wittrock); el aprendizaje como proceso de organización y representación de conocimientos en la memoria (Rumelhart y Norman); la importancia de las estrategias generales para resolver problemas (Stermberg); el aprendizaje como descubrimiento, es decir, ir trastiriendo lo aprendido a nuevas situaciones, proceso que va de la idea más simple a la más complicada para volver sobre esa misma idea de forma cada vez más compleja, en forma de espiral (Bruner); el aprendizaje como un transcurso en sucesivas fases de descontextualización: los conceptos se van haciendo más abstractos a partir de su uso en las más posibles situaciones (Bransford y Franks). Véase resumen de la temática en APARIClO, Juan José, y MaldonAdo, Antonio. "Psicología del aprendizaje y educación. Algunas consideraciones en torno a los contenidos de un módulo de psicologia educativa en la formación del profesorado", Boletín del Instituto de Ciencias de la Educación, 13 Universidad Autónoma de Madrid, diciembre de 1988, págs. 58-78.

12 Una explicación sobre las variables en ALEJos PITA, Ana, "Tecnologia educativa y comunicación en la enseñanza a distancia", en INSTITUTO DE CIENCIAS DE LA EDUCACION (UNED). El modelo español de educación superior a distancia: la UNED. Madrid, UNED, 1988, págs. 96-102.

${ }_{13}$ La bibliografia sobre teoría curricular es abundante. Como marco general: STENHOUSE, 


\section{Propuesta práctica}

Voy a fijarme en un ejemplo puntual de integración de las fuentes orales en el currículum. Concretamente en la asignatura Historia de España. Actualmente la disciplina aparece incluida en el curso tercero del Bachillerato Unificado y Polivalente ${ }^{14}$. También se ha insertado en uno de los proyectos del Plan para la Reforma de las Enseñanzas Medias, colocándola en el primer curso del ciclo correspondiente a la Enseñanza Secundaria Postobligatoria, a cursar asimismo por alumnos de dieciséis/ diecisiete años ${ }^{15}$. Los límites y finalidad de este trabajo impide explicitar pormenorizadamente el diseño curricular completo de la asignatura. Sólo señalaré algunos puntos del diseño en tanto justificarian la introducción de las fuentes orales.

Dos de los objetivos generales más fundamentales de la asignatura son: conocer las síntesis de los contenidos básicos que configuran cronológicamente el proceso histórico de España; saber interpretar hechos de ese pasado dentro del respectivo marco o modelo conceptual de la época.

Los contenidos de carácter conceptual presentarían centralmente la historia de España como un proceso complejo y continuo en el cual interconexionan diacrónica y sincrónicamente, tanto en el plano individual como colectivo, factores políticos, económicos, sociales y culturales, los cuales, a su vez, se proyectan fuera de nuestras fronteras y reciben influencias de comunidades extranjeras. Son, además, unos contenidos que incluirian las grandes líneas interpretativas y explicativas de nuestro

L., Investigación y desarrollo del curriculum, Madrid, Morata, 1984; Gimeno, J., Teoría de la enseñanza y desarrollo del curriculum. Madrid, Anaya, 1981.

${ }^{14} \mathrm{El}$ sentido de la inclusión de un caso referido a las enseñanzas medias en una revista de la UNED dedicada a la historia se relaciona con el hecho de que parte de los alumnos matriculados en esos estudios y universidad podrian dedicarse profesionalmente en su futuro a la enseñanza en el nivel secundario y parece interesante, por tanto, escoger este ejemplo, el cual se presentó como comunicación en el «Primer Encuentro de Fuentes Orales y Enseñanza de la Historia".

15 Véase MINISTERIO DE EDUCACION Y CIENCIA. Propuesta de organización de la enseñanza media reglada postobligatoria. Madrid, Ministerio de Educación y Ciencia, 1985.

La llegada al gobierno del PSOE en 1982 signíícó un replanteamiento de la educación en sus diferentes niveles. En lo que respecta a las enseñanzas medias, durante el curso 1983-1984, comienza la experiencia del primer ciclo, dentro del nivel correspondiente a la enseñanza obligatoria. La experimentación del segundo ciclo, englobado dentro de la enseñanza postobligatoria, se regula en el BOE del 4 de diciembre de 1985 y 6 de noviembre de 1986. Ambos cicłos, compuestos cada uno por dos cursos, va dirigido a alumnos comprendidos entre los catorce/quince y diecisiete/dieciocho años. 
pasado. Las construcciones históricas quedarian planteadas como cuestiones abiertas en tanto que la historiografía constituye aproximaciones a realidades inconmesurables en su total complejidad pero a las que cabe siempre la posibilidad de investigarlas con nuevas preguntas y paradigmas.

Los contenidos en su aspecto procedimental radican genéricamente en la comprensión y utilización por los alumnos de la interacción entre la teoría y los hechos concretos como principio metodológico y científico que sirve de base para la elaboración de la historia (y también de las demás ciencias naturales y humanas). Para llevar a cabo esa interacción los alumnos tendrán que emplear los instrumentos y procedimientos utilizados por los historiadores tales como el tratamiento y manejo crítico de las fuentes.

Los contenidos van agrupados en siete bloques temáticos, ordenados cronológicamente desde el primero («Las sociedades prehistóricas") hasta el séptimo ( La España del siglo xx»), aumentando el tiempo lectivo dedicado a cada uno de ellos conforme nos acercamos a nuestra época. El tiempo dedicado a "La España del siglo XX" suma, aproximadamente, treinta horas lectivas (un trimestre), dividido en las siguientes unidades didácticas:

1. "La dictadura de Primo de Rivera" (cuatro horas)

2. «La segunda república y la guerra civil» (nueve horas)

3. "La dictadura franquista" (nueve horas)

4. "La instauración de la democracia» (nueve horas).

¿Qué medios y metodología didácticas empleariamos para lograr el aprendizaje óptimo de los contenidos?

Los alumnos llegarian a aprender los contenidos curriculares tras escuchar las síntesis expositivas del profesor, leer comprensivamente el libro de texto y realizar actividades sobre material historiográfico tal como textos, esquemas, cuadros sinópticos, estadisticas, gráficos, mapas. Los contenidos procedimentales, en concreto la interconexión de un modelo teórico y los acontecimientos particulares, dentro de lo cual avanza la elaboración histórica, se conseguiría por medios de los anteriormente especificados útiles y del manejo de fuentes primarias. Para ello analizarian los hechos contenidos en la fuente y tratarian de relacionarlos con el periodo del cual proceden, estableciendo la posibilidad o no de su inclusión en alguno de los modelos definitorios de la etapa. Además, enjuiciarían la validez, fiabilidad o representatividad del documento para comprender/explicar la época o dar respuesta a los interogantes planteados. 
Cómo cada período tiene unas fuentes históricas determinadas, convendria ofrecer a los alumnos muestras de diferentes documentos primarios, entendiendo por ello cualquier tipo de material del pasado siempre que no resulte demasiado complejo para sus niveles cognoscitivos, e independientemente del soporte donde se manifieste. Porque los hechos históricos nos llegan a través de la palabra o de cualquier otro medio de elaboración humana. Las palabras pueden mostrarse en forma escrita (sobre diferentes materiales) o en forma oral. Ésta, por ejemplo, nos llega trasmitida directamente, grabada en cinta magnética, conexionada con la imagen en films o vídeo y transcrita.

Visto que el manejo de documentos resulta fundamental para la consecución de los objetivos de la asignatura, interesa apuntar las razones que justifican la inserción de las fuentes orales en el conjunto documental didáctico:

La primera reside en la propia naturaleza de los testimonios orales en tanto que constituyen parte de los materiales con los cuales abordar el análisis del pasado cercano.

Cómo la asignatura Historia de España dedica alrededor de un trimestre a estudiar el siglo xx y cómo los testimonios orales constituyen una de las fuentes para examinar aspectos históricos englobados en las citadas cuatro unidades didácticas, parece inevitable su utilización.

En tercer lugar su incorporación implica añadir a la asignatura una de las últimas innovaciones por las que atraviesa la historiografía en las últimas décadas. La agregación resulta particularmente fundamental si partimos del modelo de interconexión y fluidez que debe existir entre la escuela y la universidad $o$, podriamos decir, entre la didáctica y la investigación, entre la sociedad y la ciencia. La escuela secundaria quedaría así constituida como uno de los ámbitos donde las innovaciones de las ciencias, adaptadas, desde el punto de vista pedagógico, a las características de los escolares, serían asumidas por la base poblacional.

También utilizariamos las fuentes orales porque sintoniza con los cambios sobrevenidos dentro de la enseñanza de la historia en nuestro país durante los últimos veinte años. La enumeración de algunos de los principios asumidos por esa didáctica lleva a advertir el perfecto encaje de las fuentes orales. Por ejemplo:

a) La introducción en el aula de los materiales con los que se construye la historia. Los alumnos han comenzado a manipular documentos a partir de la generalización de publicaciones que reproducian colecciones 
de ellos. Incluso los libros de texto insertan cada vez más esa documentación.

b) La puesta en práctica de los descubrimientos de la psicologia genética, conductista y cognitiva, es decir, del conocimiento de las variables que intervienen en el aprendizaje lo cual ha fijado nuevos contenidos, metodología y estrategias didácticas.

c) La llegada de las nuevas historiografías al aula, entre ellas el retorno al tiempo corto y a lo narrativo.

d) La creación de actitudes en el alumno respecto a los hechos históricos ${ }^{16}$.

La quinta motivación legitimadora de la implantación de las fuentes orales radica en que colabora al cumplimiento de los objetivos y contenidos de la asignatura. El alumno desarrolla mayor interés y motivación hacia la historia, pues experimenta mayor implicación psicológica con la vida en el pasado. Llega a sentir directamente cómo las preguntas condicionan el conocimiento histórico. Constituye, además, una de las formas para advertir la complejidad de la realidad histórica total, al observar los variados y contradictorios discursos que genera un mismo y único hecho. Entonces, descubre más claramente la subjetividad y parcialidad de los testimonios y la necesidad de la crítica y el cotejo.

La utilización de los documentos orales sirve, como otro elemento más, para poner en práctica las teorías psicológicas sobre el aprendizaje (por ejemplo, el aprendizaje significativo). Su empleo implica interdisciplinariedad, es decir, colaboración entre diversos tipos de conocimiento (técnicas de la entrevista y muestreo, psicologia de los individuos y de las relaciones humanas, teoria de la comunicación, diferencias entre lenguajes, etc.). Desarrolla actitudes tales como la sociabilidad; creatividad; trabajo en equipo; inserción en las propias raices personales, familiares y locales; sentimientos de comprensión y tolerancia, aunque también de rechazo. Significa, una forma de ampliar el ámbito extraescolar y entrelazar la escuela con el entorno.

16 Puede seguirse algunas de las teorias y experiencias didácticas en los últimos años a través de la revista Historia 16 (Madrid) que tiene una sección titulada "Enseñar historia". También en GRUPO CRONOS (coordinación), Reflexiones sobre la enseñanza de la geografia e historia en el Reino Unido y España. Actas de las ponencias presentadas en las Jornadas sobre enseñanza de la geografia y la historia, celebradas en Salamanca (mayo de 1987). Salamanca, Instituto Universitario de Ciencias de la Educación, 1988; y en $\mathrm{C}_{\mathrm{A}}$. RRETERo, Mario; Pozo, José Ignacio; Asensio, Mikel (compiladores), La enseñanza de las ciencias sociales. Madrid, Visor, 1989. 
Expuestas las razones que creo fundamentan el engarce de las fuentes orales en la asignatura, vamos a pasar el examen de la forma o manera de su integración en el programa, sin desvirtuar significativamente el contenido temático de éste, establecido en los planes de estudio ministeriales ${ }^{17}$.

En cualquiera de las unidades didácticas correspondientes al bloque del siglo $x x$ es dable utilizar testimonios orales. A ello dedicariamos parte de las horas lectivas adjudicadas a las unidades didácticas, pues, como ya he señalado, se trata de unir las fuentes orales con los demás materiales historiográficos.

Son varias las formas por las cuales el alumno puede conocer y contactar con ellas. En principio el profesor, conociendo el contexto local del alumnado, escogería la más idónea, cómoda y operativa para el grupo.

\section{Algunas de estas formas son:}

En primer lugar a través de transcripciones de entrevistas o cuestionarios realizados por especialistas. En el Reino Unido algunos proyectos escolares siguieron esa pauta ${ }^{18}$. No sé si en España existe alguna publicación en ese sentido. Particularmente no la conozco. Por tanto, pienso que convendria imprimir algunas colecciones de entrevistas en torno a determinados centros de interés, de la misma forma que han sido editados compendios de fuentes documentales escritas, dirigidos a la ense-

17 Aqui se opta por una forma restrictiva de utilización de las fuentes orales pues el objetivo radica en obtener del alumno un conocimiento general sobre la evolución de la sociedad española y sobre las variadas formas que adopta la construcción histórica. No entro en la valoración de otras posibilidades que, dedicando más tiempo lectivo a esos documentos, implique la reducción del contenido temático de la asignatura.

Tanto en España como en el Reino Unido, Italia o Francia se han efectuado experiencias didácticas utilizando esas fuentes, existiendo bibliografia sobre el tema. El «Primer Encuentro de Fuentes Orales y Enseñanza de la Historia", recogió algunas de las realizadas en nuestro pais en los niveles educativos primarios, secundarios y superiores. Los centros de interés en torno a los cuales giraron las comunicaciones fueron: diferentes aspectos de la segunda república y la guerra civil, la revolución de 1934 en Asturias, las historia de la educación, la oposición al franquismo, la transición democrática, la historia local (Las Rozas, Elche) y regional (Canarias, Alicante).

Reseñas de las experiencias efectuadas en el Reino Unido aparecen en la revista Oral History (Essex), dentro de la sección "News". Precisamente en este país, en la conferencia del grupo History Workshop, celebrada en el Ruskin College (Oxford) en junio de 1989, se discutió sobre la introducción de las historia oral en el Currículum Nacional Escolar (Cit. en "News", Oral History, 17, 2. Essex, autum 1989, págs. 14-15.

${ }^{18}$ Según indica: ThOMPSON, Paul, «La historia oral y el historiador», Debats, 10. Barcelona, 1984, págs. 54-55. 
ñanza secundaria. Fue el mejor y más rápido modo para que pudieran llegar a las aulas de bachillerato donde se están utilizando en la actualidad con eficacia.

A la vista de las investigaciones ya efectuadas por historiadores y de las colecciones de cintas grabadas en centros documentales, parecería factible la publicación de entrevistas significativas a partir de los temas siguientes: vivencias del pueblo durante la segunda república y guerra civil; vida cotidiana y actividades femeninas; práctica política, sindical y obrera en los años treinta; exilio y emigración; guerrilla y oposición al franquismo; política internacional o relaciones exteriores. Cualquiera de estos temas puede integrarse en los contenidos del programa de la asignatura.

Otro modo estribaria en el aporte al aula, por parte del profesor, de entrevistas realizadas por especialistas, grabadas en cinta magnetofónica o en vídeo, para que los discentes las escuchasen y, analizandolas, advirtiesen también las matizaciones y mayores sugerencias que implica oir un discurso testimonial, frente al más frio y distante discurso escrito.

En tercer lugar el alumno tendría un contacto aún más directo si el mismo elabora y efectúa las preguntas a quienes vivieron el pasado. Es la manera óptima, aunque la más laboriosa para los implicados en el marco didáctico. Existen dos posibilidades.

Una reside en que el alumno, fuera de las horas lectivas, se desplace a preguntar y grabar testimonios. Un proyecto viable comprenderia en principio la elección, por parte conjuntamente de estudiantes y profesor, de un aspecto histórico y una hipótesis de trabajo a la que pudiera responder el entorno familiar o vecinal del alumno. Después, cada estudiante efectuaria una entrevista siguiendo un cuestionario previamente establecido y consensuado ${ }^{19}$, transcribiéndola a continuación. Posteriormente, como en las demás ocasiones, se llevaria a cabo en la clase la puesta en común con el material proporcionado por las entrevistas para corroborar hipótesis y verificar modelos.

19 Particularmente, basándome en la experiencia propia, creo que para la generalidad de los alumnos es difícil mantener una entrevista abierta. Ello también se indica en SEMINARIO GUILLEN ROBLES, La didáctica de la historia a través de sus fuentes: I. Fuentes orales. Málaga, Instituto de Ciencias de la Educación. Universidad de Málaga, 1985, págs. 24-25. El Seminario eligió la modalidad del cuestionario escrito, a responder por los entrevistados, en el tema "La vida cotidiana en la postguerra española", a causa de la falta de materiales para la grabación, el excesivo tiempo que se debía emplear para las transcripciones, la falta de experiencia del alumno en entrevistar, etcétera. 
La segunda posibilidad de relación inmediata consistiría en trasladar al aula a una o varias personas contemporáneas a la época que se pretende abordar. Entonces los alumnos preguntarían a la "fuente viva" sobre los acontecimientos o asuntos elegidos. Evidentemente, en la elección de esas personas intervendría el profesor. Comprendería a ciudadanos anteriormente entrevistados por él, y especialmente idóneos por su representatividad y caracteristicas personales (memoria, facilidad de palabra...).

En conclusión, puede apuntarse que resulta académica y didácticamente factible y conveniente iniciar al alumno de la asignatura Historia de España en el examen de las fuentes orales, sin desvirtuar los contenidos y características generales de la asignatura y sin imponer un trabajo extraescolar excesivo a un alumnado que tiene también, no lo olvidemos, que sacar adelante las demás disciplinas del currículum. 